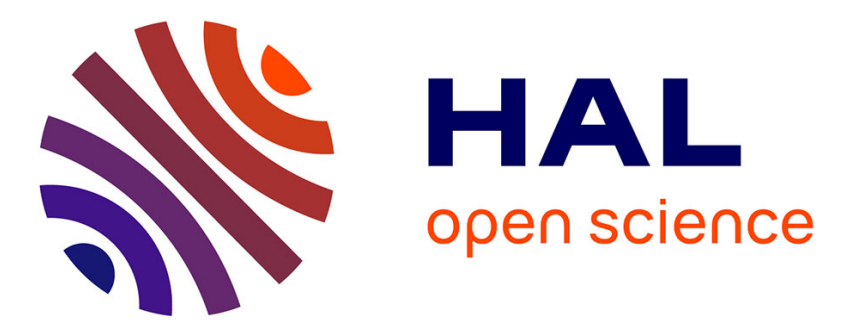

\title{
A novel stress hormone response gene in tadpoles of Xenopus tropicalis
}

Katelin A Schneider, Leena H Shewade, Nicolas Buisine, Laurent Sachs, Daniel R Buchholz

\section{- To cite this version:}

Katelin A Schneider, Leena H Shewade, Nicolas Buisine, Laurent Sachs, Daniel R Buchholz. A novel stress hormone response gene in tadpoles of Xenopus tropicalis. General and Comparative Endocrinology, 2018, 260, pp.107-114. 10.1016/j.ygcen.2018.01.006 . hal-02864265

\section{HAL Id: hal-02864265 https://hal.science/hal-02864265}

Submitted on 7 Jul 2020

HAL is a multi-disciplinary open access archive for the deposit and dissemination of scientific research documents, whether they are published or not. The documents may come from teaching and research institutions in France or abroad, or from public or private research centers.
L'archive ouverte pluridisciplinaire HAL, est destinée au dépôt et à la diffusion de documents scientifiques de niveau recherche, publiés ou non, émanant des établissements d'enseignement et de recherche français ou étrangers, des laboratoires publics ou privés. 
4 A novel stress hormone response gene in tadpoles of Xenopus tropicalis

5

6

7

8

9

10

11

12

13

14

15

16

17

18

19

20

21

22
Katelin A. Schneider ${ }^{1}$, Leena H. Shewade ${ }^{1}$, Nicolas Buisine ${ }^{2}$, Laurent M. Sachs ${ }^{2}$, Daniel R. Buchholz ${ }^{*}$

${ }^{1}$ Department of Biological Sciences, University of Cincinnati, 312 Clifton Ct, Cincinnati, OH, 45221

2UMR 7221 CNRS, Muséum National d'histoire Naturelle, Dépt. Régulation, Développement et Diversité Moléculaire, Sorbonne Universités, Paris, France

*Corresponding Author: buchhodr@ucmail.uc.edu 


\section{Abstract}

24 Previous work identified a transcribed locus, Str. 34945, induced by the frog stress hormone corticosterone (CORT) in Xenopus tropicalis tails. Because thyroid hormone had no influence on its expression, Str. 34945 was dubbed the first "CORT-only" gene known from tadpoles. Here, we examine the hormone specificity, time course of induction, tissue distribution, developmental expression profile, and genomic annotation for this transcript. We find that CORT but not aldosterone or thyroid hormone treatment induces Str. 34945 in tadpole tails and that expression of Str. 34945 achieves maximal expression within 12-24 hours of CORT treatment. Among tissues, Str. 34945 is induced to the highest degree in tail, with lesser induction in lungs, liver, and heart, and no induction in the brain or kidney. During natural metamorphosis, Str. 34945 expression in tails peaks at metamorphic climax. The location of Str. 34945 on the $X$. tropicalis genome lies between the genes ush $1 g$ (Usher syndrome 1G) and fads6 (fatty acid desaturase 6). A blast search showed that it maps to the same region on the $X$. laevis genome, but no hits were found in the human genome. Using RNA-seq data and conventional reverse transcriptase PCR and sequencing, we show that Str. 34945 is part of the 3' untranslated region of $u$ sh $1 g$. The role of ush $1 \mathrm{~g}$ in metamorphosis is not understood, but the specificity of its hormone response and its expression in tail make $U$ sh $1 g$ valuable as a marker of CORT-response gene induction independent of thyroid hormone. 


\section{Introduction}

Stress hormones induce a wide range of structural and functional changes in tissues and processes during development that are essential for survival, such as organ maturation and timing of birth (Fowden, Li, \& Forhead, 1998; Liggins, 1994). Stress hormones also mediate the effects of environmental stressors (Denver, 2009; Hu, et al., 2008; Maher, et al., 2013). When the levels of stress hormones deviate from normal during early-life development, life-long negative consequences may occur (Crespi \& Warne, 2013; Harris \& Seckl, 2011). In addition, stress hormones often do not act alone but in concert with other hormones, especially thyroid hormone (Bonett, Hoopfer, \& Denver, 2010; Terrien \& Pruinet 2013). Despite these critical roles of stress hormones and interactions with thyroid hormone in numerous tissues during development, the underlying tissue-specific molecular developmental pathways and stress hormone-regulated genes are not well characterized.

Amphibian metamorphosis is a valuable model to study the role of hormones and gene regulation due to its exquisite sensitivity and dramatic dependence on thyroid and stress hormones and ease of endocrine manipulation (Buchholz, 2015). The effects of stress hormone on development and subsequent altered stress hormone levels are conserved in tadpoles and humans (Buchholz, 2015). To begin to elucidate the genes involved in stress hormone-regulated development, we previously carried out a microarray study using Xenopus tropicalis, the African clawed frog (Kulkarni \& Buchholz, 2012). We analyzed global gene expression from tadpole tails treated with corticosterone (CORT, the frog stress hormone), thyroid hormone, CORT + thyroid hormone, or vehicle control for 18 hours. The expression of 5,432 genes was altered in response to either one or both hormones. There were 16 patterns of gene regulation due to up- or down-regulation by thyroid hormone and/or CORT. The transcribed locus Str. 34945 was among 
a small and interesting group of genes because these genes were upregulated in the presence of CORT, and thyroid hormone had no influence on their expression. We designated these genes as "CORT-only" based on this specificity of hormone induction. We chose to characterize one of these genes, Str. 34945, because it was among the most highly induced CORT-only genes. The Str. 34945 sequence is unannotated, in that it was not associated with a known gene. We examined Str. 34945 expression in key CORT target tissues and conducted a developmental expression profile through metamorphosis in the tadpole tail. We also defined the genomic annotation of Str. 34945. Knowledge of when and where this gene is expressed will greatly facilitate mechanistic studies to elucidate the role of CORT on development by virtue of having a convenient method to measure CORT action in tadpoles independent of thyroid hormone.

\section{Materials and Methods}

\section{Animal Husbandry}

Breeding was induced by priming male and female wild type Xenopus tropicalis with $20 \mathrm{U}$ of ovine luteinizing hormone (Los Angeles Biomedical Research Institute, National Hormone and Peptide Program) in the evening and boosting with 200U the next morning. The resulting tadpoles were reared at $26 \mathrm{C}$ and fed Sera Micron food twice daily with daily water changes. Tadpole tissues were harvested at developmental stages determined by Nieuwkoop and Faber (NF) (Nieuwkoop \& Faber, 1956). The use of animals in experiments was in accordance with the guidelines of the University of Cincinnati Institutional Animal Care and Use Committee (IACUC protocol \# 06-10-03-01).

2. Hormone Treatments and Tissue Harvest 
To determine hormone induction specificity, premetamorphic tadpoles at stage NF 54 were treated with $100 \mathrm{nM}$ CORT, $100 \mathrm{nM}$ aldosterone (ALDO), and $10 \mathrm{nM}$ triiodothyronine (T3), or EtOH vehicle control by addition to aquarium water for 24 hours, as done in previous studies (Kulkarni \& Buchholz, 2012; Shewade, et. al., 2017). Tails ( $\mathrm{n}=10$ per treatment) were harvested from Tricaine-anathesized animals, snap frozen, and stored at -80 until RNA isolation. For the time course, premetamorphic tadpoles at stage NF 54 were treated with $100 \mathrm{nM}$ CORT for $0,2,4$, 12, and 24 hours. A parallel experiment was done for $0,1,2,3$ and 5 days. Tails $(n=5$ per time point) were harvested and stored as above. To determine tissue distribution, premetamorphic tadpoles at stage NF 54 were treated for 24 hours with 100nM of CORT or EtOH vehicle control. The tail, lungs, liver, heart, brain, and kidney were harvested (Patmann et al, 2017) and stored as above. The sample size was $n=6$, but for smaller organs two to three individuals were pooled together to obtain enough RNA. For the developmental series, tails ( $n=6$ per stage) from tadpoles at various developmental stages throughout metamorphosis were harvested and stored as above.

\section{RNA extraction, cDNA synthesis, and qPCR}

Total RNA was extracted by using TRI Reagent RT (Molecular Research Center, Inc.) using manufacturers protocol. One ug of RNA was used in making cDNA using the manufacturer's protocol (Biotools B\&M Labs, S.A.). One $\mathrm{mL}$ of cDNA was used in $20 \mathrm{~mL}$ reactions for quantitative PCR with Taqman Universal master mix (Life Technologies) using FAM labeled primer-probe sets and run on a 7300 Real Time PCR system. The TaqMan primer probe sequences for Str. 34945 were forward: 5' CTGTAGGACACGTATTTCATGATTAAGC, reverse: 5' CAACATTAACAGGGTATGATAAAATCAATATATCTTTATTACAAAAT, and 
114 probe: 5' CCTGACGCATTTTGTG. The TaqMan primer probe sequences for the reference gene

115 ribosomal protein L8 (rpL8) were forward: 5' AGAAGGTCATCTCATCTGCAAACAG,

116 reverse: 5' CTTCAGGATGGGTTTGTCAATACGA, and probe: 5'

117 CAACCCCAACAATAGCT. The reaction conditions for were $50^{\circ} \mathrm{C} 2 \mathrm{~min}, 95^{\circ} \mathrm{C} 10 \mathrm{~min}$, then

11840 cycles of $95^{\circ} \mathrm{C} 10 \mathrm{sec}, 60^{\circ} \mathrm{C} 1 \mathrm{~min}$. Relative quantification was carried out using the delta

119 delta Ct method (Livak \& Schmittgen, 2001).

120

121 4. Genomic characterization of Str.34945

122 RNA-seq analysis:

123 The datasets used for genome-wide profiling of gene expression were from (Buisine, et. al., 124 2015) and followed the same workflow. Briefly, saturating amounts (> 35-40 M reads) of high 125 quality SOLiD strand specific reads were mapped on the Xenopus tropicalis genome (v4.1) with 126 the Bioscope pipeline, run with stringent parameters. Only reads mapping at a single genomic

127 location were kept. Read density profiles were normalized over the lowest sequencing depth and 128 displayed on a genome browser. A total of five libraries were processed, corresponding to a 129 collection of larval (tailfin, limb buds) and adult (intestine, kidney, brain) and tissues. Although a 130 more recent version of the genome assembly is available, our use of version 4.1 has no impact on 131 the results while we benefit from the high quality annotation described in Buisine et al. (Buisine, 132 et. al., 2015). Importantly, this annotation is based on the RNA-PET technology, which is

133 designed to specifically capture the $5^{\prime}$ (and 3' end, to some extent) end of transcripts, thus

134 providing a higher resolution map of transcript boundaries.

136 Conventional reverse transcriptase PCR analysis: 
RNA and cDNA from NF54 tails of tadpoles treated with and without $100 \mathrm{nM} \mathrm{CORT}$ or vehicle

138 for 24 hrs. were used in PCR (Takara Bio Inc., MountainView, CA). Primer sequences for Str.

13934945 and coding regions of $u s h 1 g$ and fads6 are listed in Table 1. All PCR products were run

140 on $1 \%$ agarose gels and visualized with ethidium bromide. The results were independently

141 obtained 3 times. Controls with no reverse transcriptase produced no bands (data not shown).

142 The PCR product obtained from the fourth exon of $u s h 1 g$ to Str. 34945 was spin column purified

143 and sequenced. Genome analyses were conducted using Xenbase (http://www.xenbase.org/,

144 RRID:SCR_003280).

146 5. Statistical analysis

147 Analysis of variance (ANOVA) was performed to identify significant differences in Str. 34945

148 expression among developmental stages and hormone treatments using JMP statistical analysis

149 software (JMP Pro 12). Pair-wise comparisons were done using the Tukey-Kramer post-hoc test,

150 and a $p$-value less than 0.05 was considered to be statistically significant. All error bars are

151 reported as standard error of the mean.

152

153 Results

154 1. Hormone Specificity

155 To confirm the specificity of hormone regulation for Str. 34945, as suggested by our previous

156 microarray (Kulkarni \& Buchholz, 2012), we measured Str. 34945 expression in the tail of $X$.

157 tropicalis after CORT (100nM), aldosterone (100nM), and triiodothyronine (T3, 10nM)

158 treatments. Expression of Str. 34945 mRNA was induced exclusively by CORT and not

159 aldosterone or T3 (Figure 1). Such verification was important because analysis of another 
putative "CORT-only" gene from the microarray (pckl) revealed some induction by thyroid

161 hormone (Kulkarni \& Buchholz, 2012).

162

163

2. Time course of Str. 34945 induction by CORT in tails

164 To determine the optimal treatment time of CORT induction of Str. 34945 expression, we measured Str. 34945 expression after 0, 2, 4, 12, and 24 hours and 0, 1, 2, 3, and 5 days of 100nM CORT treatment in tadpole tails. Str. 34945 mRNA levels remained low at 0, 2, and 4 hours and then maintained a 6- to 8-fold induction from 12 hours to 5 days (Figure 2). Based on the results obtained, we chose to conduct all further hormone treatments for 24 hours.

3. Tissue Distribution

171 We next examined which organs other than tail exhibited induction of Str. 34945 expression

172 after exogenous CORT treatment. We examined Str. 34945 mRNA levels in the tail, lungs, liver,

173 heart, brain, and kidney. Str. 34945 expression significantly increased in the tail by 7 -fold, lungs

174 by 3 -fold, and liver and heart by 2-fold (Figure 3). Exogenous CORT did not significantly

175 increase Str. 34945 mRNA levels in the brain and kidney (Figure 3).

176

177 4. Developmental Profile of Str. 34945

178 Because Str. 34945 expression is regulated by CORT, we expected that Str. 34945 expression

179 levels would follow the developmental profile of CORT level in plasma, which peaks at the

180 climax of metamorphosis (Hatey \& Jaudet, 1984). We measured Str. 34945 in the tails of

181 tadpoles throughout tadpole development and found that Str. 34945 mRNA began to rise at

182 prometamorphosis (NF 58) and peaked at metamorphic climax (NF 62) (Figure 4). 
184 5. Genomic analysis of Str. 34945

185 To determine the identity of Str. 34945, we began by examining RNA-seq data from larval and

186 adult tissues. Read counts achieved peaks in the coding exons of $u s h 1 g$, and read counts at the $3^{\prime}$

187 untranslated region extended beyond the annotated portion of $u s h l g$ and seemed to represent a

188 continuous transcript through a length encompassing all of Str. 34945 (Figure 5). Essentially no

189 expression of fads 6 was detected in the tissues analyzed.

190 To support the RNA-seq data that Str.34945 is a portion of the 3' untranslated region of

191 ush $1 g$, we measured tail expression levels of ush $1 g$ and fads6 after $100 \mathrm{nM} \mathrm{CORT} \mathrm{treatment}$

192 (Figure 6A). We found that each exon of ush $1 g$ was upregulated by CORT, as is Str. 34945.

193 Fads6 was not well expressed in tail nor was it upregulated by CORT. As a positive control for

194 the fads6 PCR primers, we observed expression of fads6 in tadpole brain, which was not

195 regulated CORT (data not shown).

196 To confirm that Str. 34945 is part of the 3' untranslated region of $u$ sh $1 g$, we designed

197 PCR primers to amplify the region between the 3' end of the current genomic annotation of

198 ush $1 g$ and the $5^{\prime}$ end of Str.34945 using conventional reverse transcriptase quantitative PCR. A

199 PCR product of the expected length was obtained and sequenced (Fig. 6B). The sequence

200 matched the $X$. tropicalis genome version 4.1 exactly (Hellsten et al., 2010), confirming that Str.

20134945 is a part of the $u s h \lg$ transcript.

203 Discussion

204 Corticosteroid-dependent developmental processes are complex, and there is still much to 205 learn about the signaling mechanisms and effects of stressors on development (Crespi \& Warne, 
2013; Kulkarni \& Buchholz, 2014). To add to our current understanding, we characterized the endogenous expression and genomic annotation of a transcribed locus upregulated by CORT in tadpoles, Str. 39345, which was originally identified in a microarray analysis of hormone regulated genes in tadpole tails (Kulkarni \& Buchholz, 2012).

Our interest in this gene derived from the novel pattern of hormone induction. The only previously known CORT-response gene in tadpoles was the transcription factor klf9, but klf9 is also regulated independently by thyroid hormone and synergistically with thyroid hormone and CORT (Bagamasbad et al., 2012; Bonett et al., 2009). CORT is also known to alter deiodinase activity affecting thyroid hormone signaling in a tissue-, stage-, and condition-specific manner, but such altered activity may not be due to CORT action on deiodinase genes at the transcriptional level (Bonett et al., 2010; Martinez-deMena, et al., 2016; Van der Geyten \& Darras, 2005). Here, we confirmed the microarray study that CORT induced Str. 34945 expression with no influence from thyroid hormone. Str.34945 is also not induced by $100 \mathrm{uM}$ ALDO, indicating it is a bone fide "CORT-only" gene. This gene may be a direct response gene as its induction starts to increase between 4 and 12 hours, though we have not confirmed this with co-treatment of CORT with cycloheximide or identified a glucocorticoid hormone response element. In addition, as expected for a CORT-response gene, expression of Str.34945 peaked at metamorphic climax when endogenous CORT also reaches its peak (Glennemeier, Denver, 2002; Leloup \& Buscaglia 1977)

Interestingly, even though most if not all tissues express glucocorticoid receptors including brain, tail, and lung (Shewade et al., 2017), only four of the six tissues tested induced Str.34945 in response to CORT, namely tail, lung, liver, heart. The tail gave the greatest degree of induction at about 7-fold, and the fact that thyroid hormone does not affect its expression 
makes this transcript unique for studies in CORT action and glucocorticoid endocrine disruption. Indeed, a recent study examined the expression of several genes originally identified in our previous microarray study for use in an ex vivo tail tip assay for screening glucocorticoid disrupting chemicals, though they did not examine Str. 34945 and did not independently verify that the genes they chose were not inducible by thyroid hormone (Chen, et. al., 2017).

A further goal for this research was to determine the identity of Str. 34945 beyond a "transcribed locus" as indicated in the Unigene database. The Str. 34945 sequence is derived 236 from six expressed sequence tags (ESTs) from testis and brain of adult frogs and maps to an 237 unannotated genomic region between $u s h 1 g$ and fads 6 in the $X$. tropicalis genome. The largest 238 open reading frame of the 2053bp nucleic acid sequence of Str. 34945 is 90 amino acids and 239 gave no BLAST hits to the human and mouse protein databases. Thus, Str.34945 does not appear 240 to code for a protein. However, Str. 34945 does have a polyA tail suggesting it may be part of a 241 protein coding transcript.

We next determined if Str. 34945 could be part of the untranslated region of an adjacent

243 gene, i.e., ushlg or fads6, or could be its own transcript. RNA-seq data suggested that Str. 34945 244 could be part of a large 3' untranslated region of $u s h 1 g$, giving no evidence that it could be part 245 of the 5' untranslated region of fads6. If Str. 34945 were part of the $u s h 1 g$ transcript, then $u s h 1 g$ 246 should be CORT-inducible, which is indeed what we found. However, it was possible that $u s h 1 g$ 247 and Str. 34945 were two different CORT-induced transcripts. We reasoned that if the 248 unannotated region between $u s h 1 g$ and Str. 34945 were both part of a single transcript, we 249 should be able to amplify it using reverse transcriptase PCR. Indeed, we obtained a PCR product 250 of the expected length, and the sequence matched the genomic region between ush $1 g$ and Str. 
34945 enabling us to conclude that Str. 34945 corresponds to a portion of the 3 ' untranslated region of ushlg.

Our PCR sequence matched the $X$. tropicalis genome sequence v4.1 exactly with minor differences compared to $X$. tropicalis genome v7.1. Interestingly, the sequence of one of the ESTs comprising Str. 34945, i.e., CX896217, matches genome v7.1 exactly, suggesting potential strain differences in this region of the $X$. tropicalis genome. Using BLAST, the non-repetitive portions of Str. 34945 hit in the unannotated regions of the $X$. laevis genome just 5 ' of $u$ sh $1 g$ in chromosome9_10L/S (Session et al., 2016). Also, RepeatMasker (Smit et al. 2013) found a DNA transposon (hobo-Activator) in $\sim 280$ bases of the Str. 34945 sequence and there was a DNA transposon at the homologous site in X. laevis. No ESTs are known from the coding region of ushlg in X. tropicalis, but a Unigene from X. laevis is known, X1.81120, which is comprised of three sequences from two cDNA libraries from gastrula and NF53 tadpole whole body. Using BLAST to compare the non-repetitive region of Str. 34945 against the human and mouse genomes gave no hits. However, synteny including at least four genes with $u s h 1 g$ was observed between the human and frog genomes (Fig. 6C). Human and frog share the intron exon structure for $u s h 1 g$, and exon 4 in both species begins with the sequence "ATAA", which encodes the STOP codon, followed by long 3' untranslated regions. The 3' untranslated region of human ush $1 \mathrm{~g}$ is about 2000bp compared to 3309bp of Xenopus encompassing Str. 34945, but Xenopus transcripts tend to have long 3' untranslated regions (Buisine, et al., 2015). Human expression from EST data for ush $1 g$ is in testis, ovary, endometrium, skin, esophagus, adrenal (Uhlen et al., 2015).

The potential role of $u s h l g$ in metamorphosis is not known, as nothing is known about this gene in frogs other than its representation in the Unigene expression database and expression 
in Xenopus larval photoreceptor cells (Maerker et al., 2008; Sahly et al., 2012). Ush1g is a protein coding gene that is essential in the development and maintenance of the visual and auditory systems in mammals (Reiners, et al., 2006; Sorusch et al., 2017). Also known as SANS (scaffold protein containing ankyrin repeats and SAM domain), ush $1 g$ is a structural protein that associates with an intracellular protein complex near the base of cilia in photoreceptors and cochlear hair cells (Adato et al., 2005). Mutations in this gene are associated with Usher's syndrome type $1 \mathrm{G}$, which is an autosomal recessive disease that results in congenital sensorineural deafness and retinitis pigmentosa (Weil et al., 2003). Given this previous knowledge of the function and expression of $u s h 1 g$, insight into potential roles in tail resorption and other events of metamorphosis remain obscure.

In summary, we have characterized the first CORT-only response gene, $u s h l g$, in tadpoles with respect to time course of induction, tissue and developmental expression profiles, and genomic annotation. This gene will be useful in future studies of CORT action during development and metamorphosis and additional studies will be required to understand its developmental roles.

\section{Acknowledgements}

This work was supported by University of Cincinnati Department of Biological Sciences

Wieman-Wendel-Benedict grant to K.A.S. and is in partial fulfillment of a Master of Science degree for K.A.S. This work was supported by the "Centre National de la Recherche Scientifique" and the "Muséum National d'Histoire Naturelle" to N.B. and L.M.S. 


\section{References Cited}

Adato, A., Michel, V., Kikkawa, Y., Reiners, J., Alagramam, K. N., Weil, D., ... Petit, C. (2005). Interactions in the network of Usher syndrome type 1 proteins, 14(3), 347-356. http://doi.org/10.1093/hmg/ddi031

Bagamasbad, P., Ziera, T., Borden, S. A., Bonett, R. M., Rozeboom, A. M., Seasholtz, A., \& Denver, R. J. (2012). Molecular Basis for Glucocorticoid Induction of the Krüppel-Like Factor 9 Gene in Hippocampal Neurons, 153(November), 5334-5345. http://doi.org/10.1210/en.2012-1303

Bonett, R. M., Hoopfer, E. D., \& Denver, R. J. (2010). Molecular mechanisms of corticosteroid synergy with thyroid hormone during tadpole metamorphosis. General and Comparative Endocrinology, 168(2), 209-219. http://doi.org/10.1016/j.ygcen.2010.03.014

Bonett, R. M., Hu, F., Bagamasbad, P., \& Denver, R. J. (2009). Stressor and glucocorticoiddependent induction of the immediate early gene kr??ppel-like factor 9: implications for neural development and plasticity. Endocrinology, 150(4), 1757-1765. http://doi.org/10.1210/en.2008-1441

Buchholz, D. R. (2015). More similar than you think : Frog metamorphosis as a model of human perinatal endocrinology. Developmental Biology, 1-8. http://doi.org/10.1016/j.ydbio.2015.02.018

Buisine, N., Ruan, X., Bilesimo, P., Grimaldi, A., \& Alfama, G. (2015). Xenopus tropicalis Genome Re-Scaffolding and Re-Annotation Reach the Resolution Required for In Vivo ChIA-PET Analysis, 1-27. http://doi.org/10.1371/journal.pone.0137526

Chen, X., Li, Y., Zhu, M., \& Qin, Z. (2017). ScienceDirect An ex vivo assay for screening 
glucocorticoid signaling disruption based on glucocorticoid-response gene transcription in Xenopus tails. Journal of Environmental Sciences, 1-9. http://doi.org/10.1016/j.jes.2017.05.017

Crespi, E. J., \& Warne, R. W. (2013). Integrative and Comparative Biology Environmental Conditions Experienced During the Tadpole Stage Alter Post-metamorphic Glucocorticoid Response to Stress in an Amphibian, 1-13. http://doi.org/10.1093/icb/ict087

Denver, R. J. (2009). Stress hormones mediate environment-genotype interactions during amphibian development. General and Comparative Endocrinology, 164(1), 20-31.

Fowden, A. L., Li, J., \& Forhead, A. J. (1998). Glucocorticoids and the preparation for life after

Harris, A., \& Seckl, J. (2011). Hormones and Behavior Glucocorticoids, prenatal stress and the

Hatey, L., \& Jaudet, J. (1984). Variations in Aldosterone and Corticosterone Plasma Levels during Metamorphosis in Xenopus laevis Tadpoles, 65, 59-65.

Hellsten, U., Harland, R. M., Gilchrist, M. J., Hendrix, D., Jurka, J., Blitz, I. L., ... Amaya, E. (2010). charge recombination reaction, it was proposed that structural changes occur- ring in response to electron transfer decrease the free energy gap between $\mathrm{P}+$ and $\mathrm{Q}, 328($ April), 633-637.

Hu, F., Crespi, E. J., \& Denver, R. J. (2008). Programming Neuroendocrine Stress Axis Activity by Exposure to Glucocorticoids during Postembryonic Development of the Frog, Xenopus 
Kulkarni, S. S., \& Buchholz, D. R. (2012). Beyond synergy: Corticosterone and thyroid hormone have numerous interaction effects on gene regulation in Xenopus tropicalis tadpoles. Endocrinology, 153(11), 5309-5324. http://doi.org/10.1210/en.2012-1432

Kulkarni, S. S., \& Buchholz, D. R. (2014). Corticosteroid signaling in frog metamorphosis, (March 2016). http://doi.org/10.1016/j.ygcen.2014.03.036

Liggins, G. C. (1994). The Role of Cortisol in Preparing the Fetus for Birth *.

Livak, K. J., \& Schmittgen, T. D. (2001). Analysis of Relative Gene Expression Data Using RealTime Quantitative PCR and the $2 \triangle \bigotimes \bigotimes C T$ Method, 408, 402-408.

$$
\text { http://doi.org/10.1006/meth.2001.1262 }
$$

Maerker, T., Wijk, E. Van, Overlack, N., Kersten, F. F. J., Mcgee, J., Goldmann, T., ... Wolfrum, U. (2008). A novel Usher protein network at the periciliary reloading point between molecular transport machineries in vertebrate photoreceptor cells, 17(1), 71-86. http://doi.org/10.1093/hmg/ddm285

Maher, J. M., Werner, E. E., Denver, R. J., \& Maher, J. M. (2013). Stress hormones mediate predator- induced phenotypic plasticity in amphibian tadpoles. http://doi.org/10.5061/dryad.1kf76

Martinez-deMena, R., Calvo, R. M., Garcia, L., \& Obregon, M. J. (2016). Effect of glucocorticoids on the activity, expression and proximal promoter of type II deiodinase in rat brown adipocytes. Molecular and Cellular Endocrinology, 428, 58-67. http://doi.org/10.1016/j.mce.2016.03.021

Reiners, J., Nagel-wolfrum, K., Ju, K., Wolfrum, U., \& Ma, T. (2006). Molecular basis of human 
Usher syndrome : Deciphering the meshes of the Usher protein network provides insights into the pathomechanisms of the Usher disease, 83. http://doi.org/10.1016/j.exer.2005.11.010

Sahly, I., Dufour, E., Schietroma, C., Michel, V., Bahloul, A., Perfettini, I., ... Petit, C. (2012). Localization of Usher 1 proteins to the photoreceptor calyceal processes, which are absent from mice, 199(2), 381-399. http://doi.org/10.1083/jcb.201202012

Session, A. M., Uno, Y., Kwon, T., Chapman, J. A., Toyoda, A., Takahashi, S., ... Matsuda, Y. (2016). Genome evolution in the allotetraploid frog Xenopus laevis. Nature, 538(7625), 336-343. http://doi.org/10.1038/nature19840

Shewade, L. H., Schneider, K. A., Brown, A. C., \& Buchholz, D. R. (2017). General and Comparative Endocrinology In-vivo regulation of Krüppel-like factor 9 by corticosteroids and their receptors across tissues in tadpoles of Xenopus tropicalis. General and Comparative Endocrinology, 248, 79-86. http://doi.org/10.1016/j.ygcen.2017.02.007

Sorusch, N., Bauß, K., Plutniok, J., Samanta, A., Knapp, B., Nagel-wolfrum, K., \& Wolfrum, U. (2017). Characterization of the ternary Usher syndrome SANS / ush2a / whirlin protein complex, 26(6), 1157-1172. http://doi.org/10.1093/hmg/ddx027

Uhlen, M., Fagerberg, L., Hallstrom, B. M., Lindskog, C., Oksvold, P., Mardinoglu, A., ... Ponten, F. (2015). Tissue-based map of the human proteome. Science, 347(6220), 12604191260419. http://doi.org/10.1126/science.1260419

Van der Geyten, S., \& Darras, V. M. (2005). Developmentally defined regulation of thyroid hormone metabolism by glucocorticoids in the rat. Journal of Endocrinology, 185(2), 327336. http://doi.org/10.1677/joe.1.05974 
Weil, D., El-Amraoui, A., Masmoudi, S., Mustapha, M., Kikkawa, Y., Lainé, S., ... Petit, C. (2003). Usher syndrome type I G (USH1G) is caused by mutations in the gene encoding SANS, a protein that associates with the USH1C protein, harmonin. Human Molecular Genetics, 12(5), 463-471. http://doi.org/10.1093/hmg/ddg051

390 Table 1. Primer table for conventional PCR.

\begin{tabular}{|c|c|c|l|l|c|}
\hline Gene & Exon span & Primer Name & Forward Primer (5'-3') & Reverse Primer (5'-3') & Product (bp) \\
\hline rpl8 & E3-6 & DRB21b/22b & $\begin{array}{l}\text { CGTGGTGCTCCTCTTG } \\
\text { CCAAG }\end{array}$ & $\begin{array}{l}\text { GACGACCAGTACGAC } \\
\text { GAGCAG }\end{array}$ & 577 \\
\hline ush1g & E1-E2 & DRB325/326 & $\begin{array}{l}\text { AGAGACCTGAAC } \\
\text { TCGCCAGA }\end{array}$ & $\begin{array}{l}\text { GGTGGGAAACAT } \\
\text { TGTGGCAC }\end{array}$ & 525 \\
\hline ush1g & E3-E4 & DRB333/334 & $\begin{array}{l}\text { CTGGGCGAGGAA } \\
\text { GTGAAGTT }\end{array}$ & $\begin{array}{l}\text { CGGTAGTCTTGGC } \\
\text { AGCAGAA }\end{array}$ & 528 \\
\hline $\begin{array}{c}\text { ush1g / } \\
\text { Str. 34945 }\end{array}$ & E4- & $\begin{array}{l}\text { KSForward/ } \\
\text { KSReverse }\end{array}$ & $\begin{array}{l}\text { TTCTGCTGCCAAG } \\
\text { ACTACCG }\end{array}$ & $\begin{array}{l}\text { CATGTCCCCTTTA } \\
\text { GATCCCCC }\end{array}$ & 1404 \\
\hline Str. 34945 & - & SSK34/33 & $\begin{array}{l}\text { ATCGGGTCAAGT } \\
\text { CGGTATTATCC }\end{array}$ & $\begin{array}{l}\text { AGGGCAGTGGAA } \\
\text { ACAGTAACTCAC }\end{array}$ & 274 \\
\hline fads6 & E1-E4 & DRB327/328 & $\begin{array}{l}\text { AAAAAGAGCAGC } \\
\text { TGGTGGGA }\end{array}$ & $\begin{array}{l}\text { GAGGAACACGCA } \\
\text { CACAATGG }\end{array}$ & 561 \\
\hline
\end{tabular}

\section{Figure legends}

395 Figure 1. Hormone induction specificity of Str. 34945 in tadpole tails. Premetamorphic tadpoles

396 were treated with vehicle control, $100 \mathrm{nM}$ CORT (corticosterone), $10 \mathrm{nM} \mathrm{T3}$ (triiodothyronine),

397 and $100 \mathrm{nM}$ ALDO (aldosterone) for $24 \mathrm{hrs}$, followed by tail RNA extraction and RT-qPCR for 398 Str. 34945 and the housekeeping gene $r p L 8$. Str. 34945 was induced only by CORT and not by

399 T3 or ALDO. Bars show mean mRNA levels relative to the housekeeping gene $r p L 8$. Error bars 
indicate SEM. The letters above the error bars indicate significance groups among treatments based on Tukey's honest significant difference test ( $\mathrm{p}<0.05, \mathrm{n}=10$ tails per treatment group).

Figure 2. Time course of Str. 34945 induction by CORT in tadpole tails. Premetamorphic tadpoles were treated with vehicle control and $100 \mathrm{nM}$ CORT (corticosterone) for (A) $0,2,4,12$, 24 hours and (B) 0, 1, 2, 3, 5 days, followed by tail RNA extraction and RT-qPCR for Str. 34945 and the housekeeping gene rpL8. Maximal expression of Str. 34945 was achieved by 12 hrs. and was maintained for 5 days. Bars show mean mRNA levels relative to the housekeeping gene $r p L 8$. Error bars indicate SEM. The letters above the error bars indicate significance groups among treatments based on Tukey's honest significant difference test $(\mathrm{p}<0.05, \mathrm{n}=5$ tails per treatment group).

Figure 3. Tissue expression profile of Str. 34945 in tadpoles. Premetamorphic tadpoles were treated with vehicle control and $100 \mathrm{nM}$ CORT (corticosterone) for 24 hrs., followed by RNA extraction from harvested tails, lungs, livers, hearts, brains, and kidneys and then by RT-qPCR for Str. 34945 and the housekeeping gene $r p L 8$. Str. 34945 was most highly induced in tails, moderately induced in lungs, liver, and heart, and not induced in brain and kidney. Bars show the mean mRNA levels relative to the housekeeping gene $r p L 8$. Error bars represent SEM. The letters above the error bars indicate significance groups among treatments based on Tukey’s honest significant difference test $(\mathrm{p}<0.05, \mathrm{n}=6$ for tails and brains, $\mathrm{n}=5$ for livers and kidneys, $\mathrm{n}=4$ for lungs and hearts per treatment group). 
Figure 4. Developmental expression profile of Str. 34945 in tadpole tails throughout the larval period. Tails from tadpoles at the indicated Nieuwkoop and Faber (NF) stages (Nieukoop \& Faber, 1956) were harvested, followed by tail RNA extraction and RT-qPCR for Str. 34945 and the housekeeping gene $r p L 8$. The expression of Str. 34945 increased significantly at metamorphic climax (NF 62-64). Bars show the mean mRNA levels relative to the housekeeping gene $r p L 8$. Error bars represent SEM. The letters above the error bars indicate significance groups among stages based on Tukey's honest significant difference test $(\mathrm{p}<0.05, \mathrm{n}=6$ for tails).

Figure 5. RNA-seq expression data for Str. 34945 in larval and adult organs. The data for RNAseq, as well as Gurdon EST clusters, cloned cDNA, and MNHN annotation (Muséum national d'Histoire naturelle), are shown mapped onto Scaffold 504 of the Xenopus tropicalis genome v4.1 in the region of ush $1 g$ and fads6. Exon-intron structures for ush $1 g$ (4 exons) and fads6 (6 exons) are shown with blue boxes on the MNHN annotation track. The location of Str. 34945 is shown with a large grey box in the Gurdon EST cluster track (the smaller grey boxes are Gurdon ESTs for fads 6 ) and with orange boxes on the cloned cDNA track. Read count peaks indicated by the orange histograms are seen predominantly underneath the exons of ush $1 g$ and not fads6. The read count peaks in ush $1 g$ extend beyond the annotated region of exon 4 to the 3 ' end of Str. 34945, suggesting a single continuous transcript including ushlg and Str. 34945.

Figure 6. Str. 34945 is part of the 3' untranslated region of ush 1 . A. Premetamorphic tadpoles were treated with vehicle control and $100 \mathrm{nM} \mathrm{CORT} \mathrm{(corticosterone)} \mathrm{for} 24$ hrs., followed by tail RNA extraction and conventional PCR for the indicated regions of $u s h 1 g$, Str. 34945, fads 6 and 
$445 r p L 8 . \mathrm{E}=$ exon. CORT induction was observed in ushlg and Str. 34945 and not fads6. RpL8 was 446 used as a quality control. B. Current annotation of Str. 34945 from Xenbase is shown above the 447 annotation established by this study. The 5' and $3^{\prime}$ untranslated regions of $u s h 1 g$ are dark, and the 448 ushlg exons are orange. The green bar indicates the PCR product, from tail cDNA, whose 449 sequence matched the genomic sequence. C. Synteny analysis of the frog and human genomes 450 indicate that the frog and human $u s h 1 g$ are homologs and that the genomic location of $u s h 1 g$ is 451 conserved in frogs and humans. The four genes are otop2 (otopterin 2), ush 1 , fads6, and fdxr 452 (ferredoxin reductase).

453 


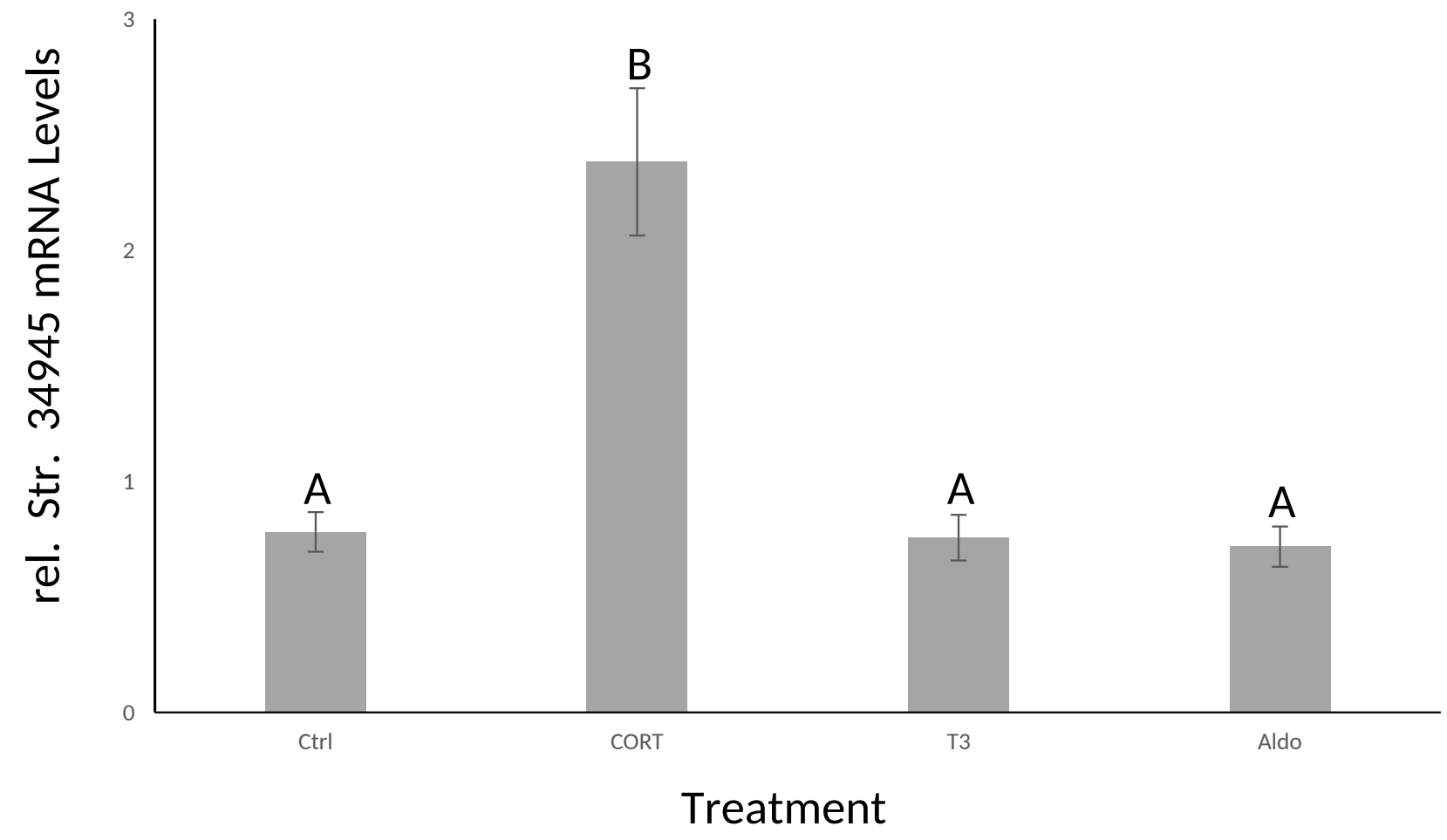



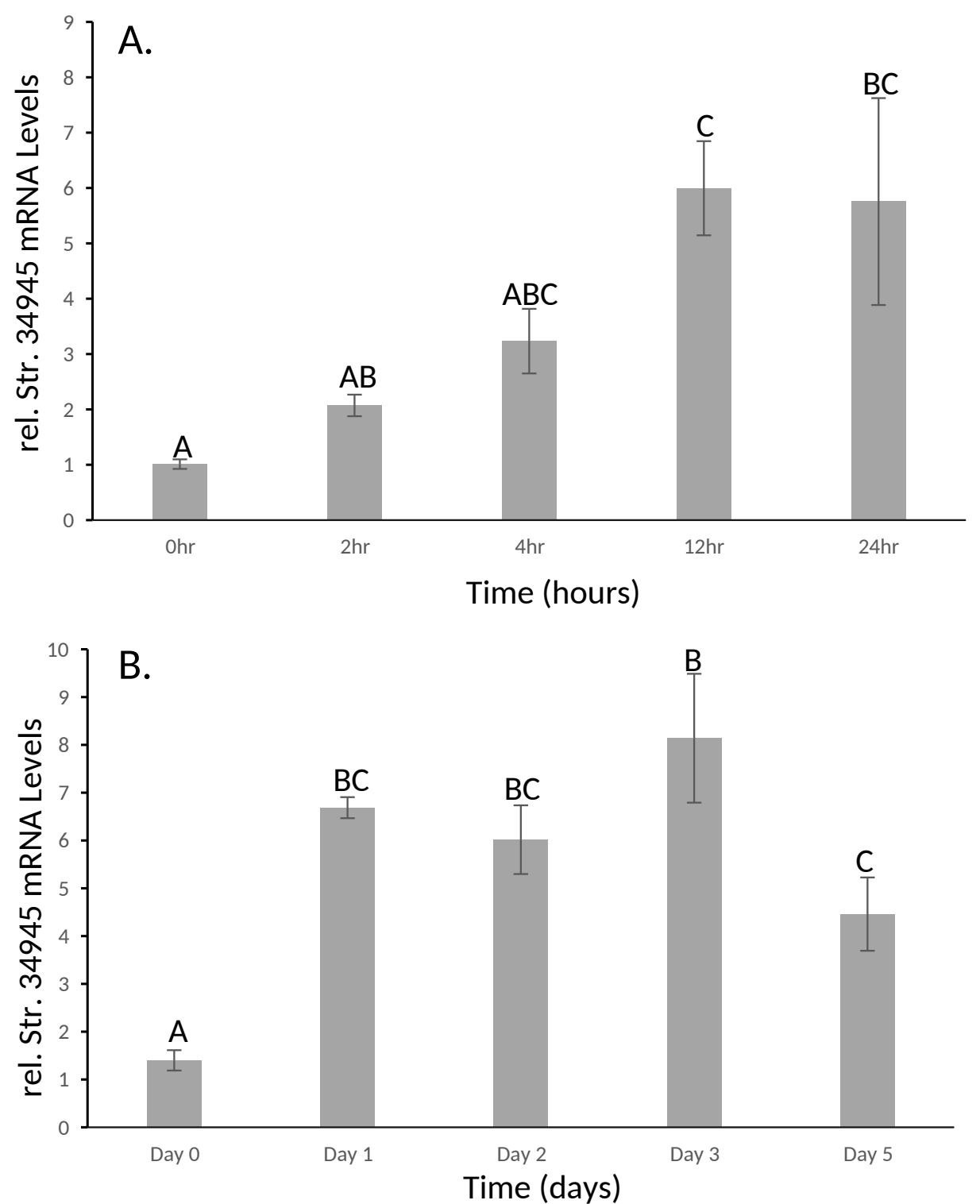


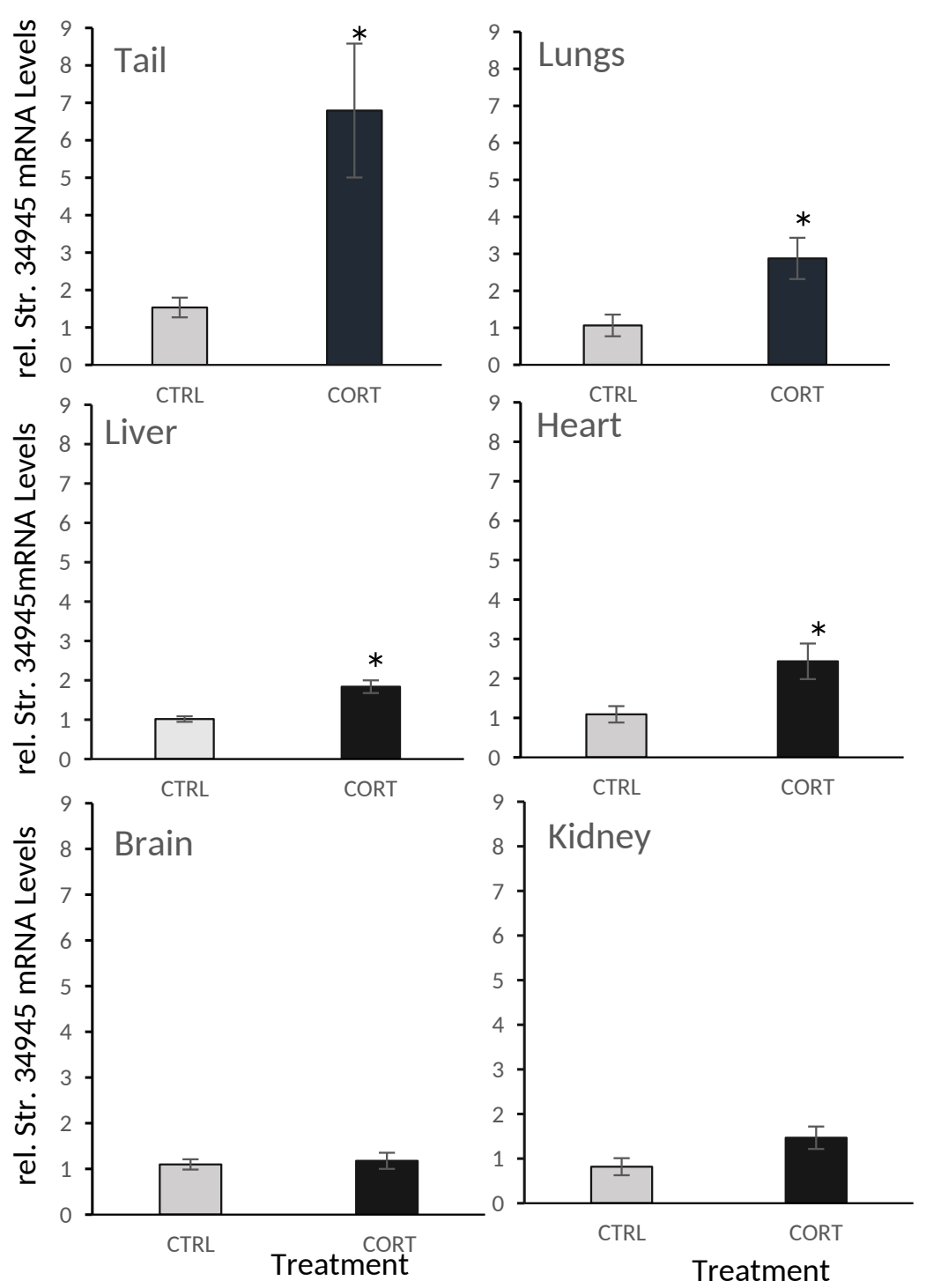




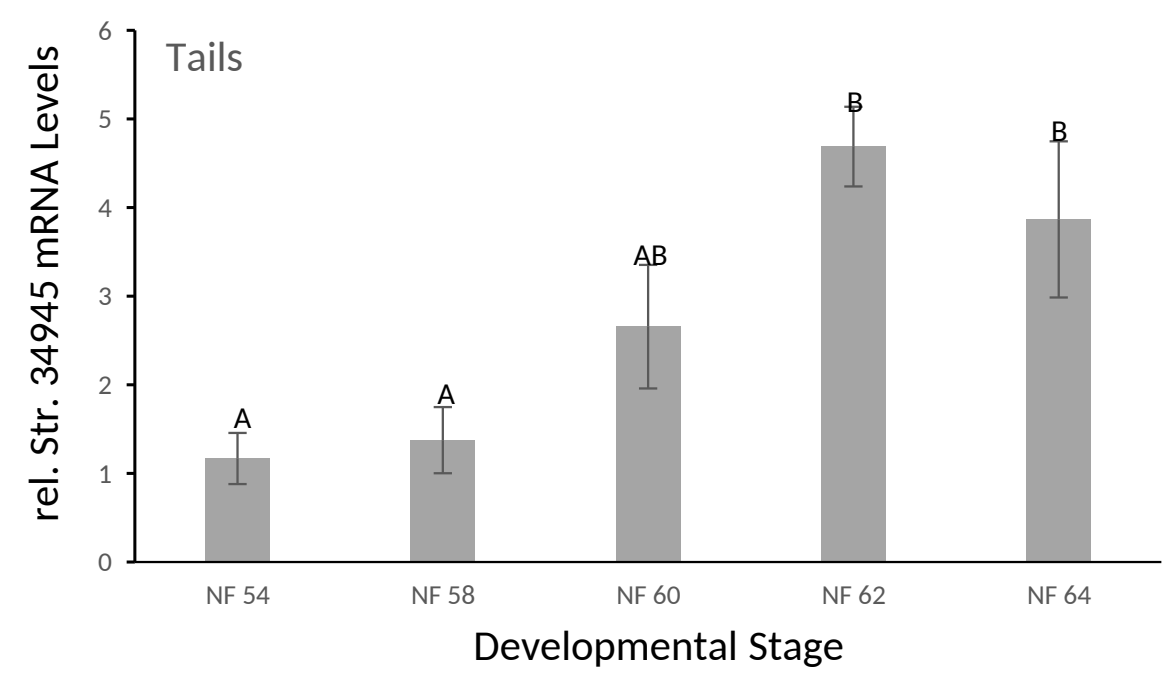


scaffold_504

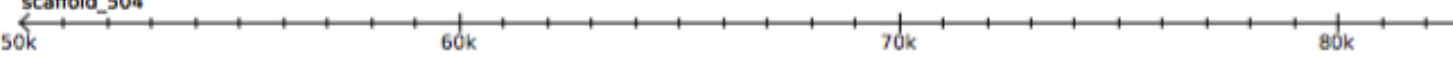

GURDON EST clusters

Cloned eDNA

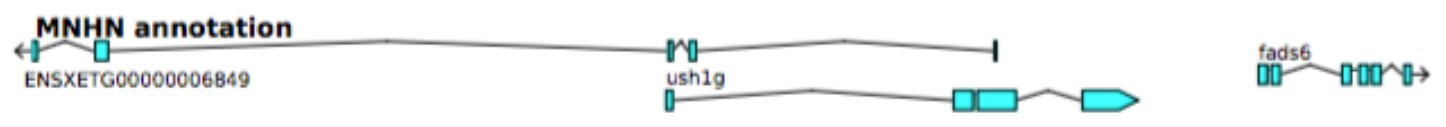

Larval limb

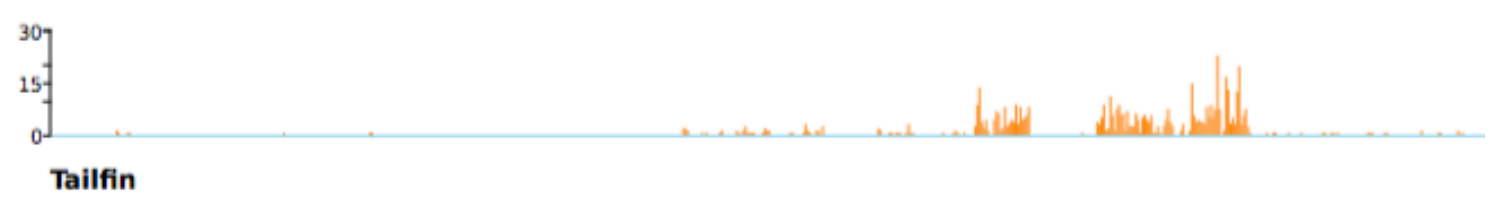

$$
\left.{ }^{30}\right]
$$

Adult intestine

$$
\left.{ }^{30}\right]
$$

Adult kidney

$$
\text { } 307
$$

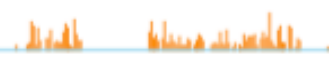

Adult brain

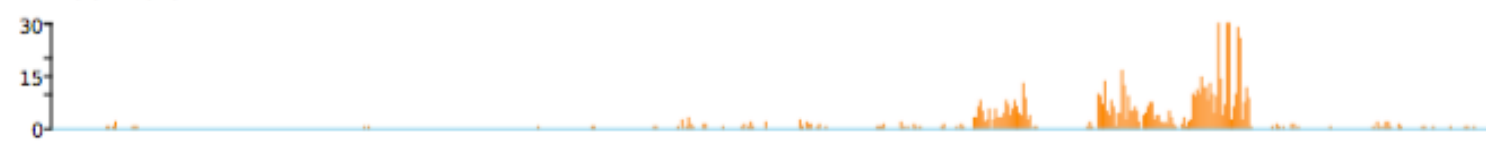


A. Primer Name

Ctrl CORT

ush1g E1-3

ush1g E3-4

Str. 34945

fads6 E1-3

$r p L 8$

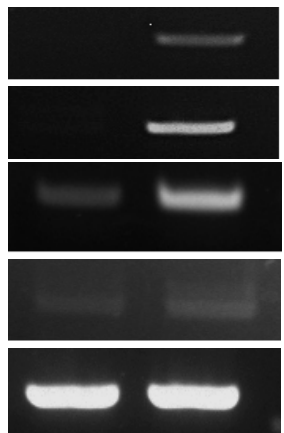

B. Old and new annotation
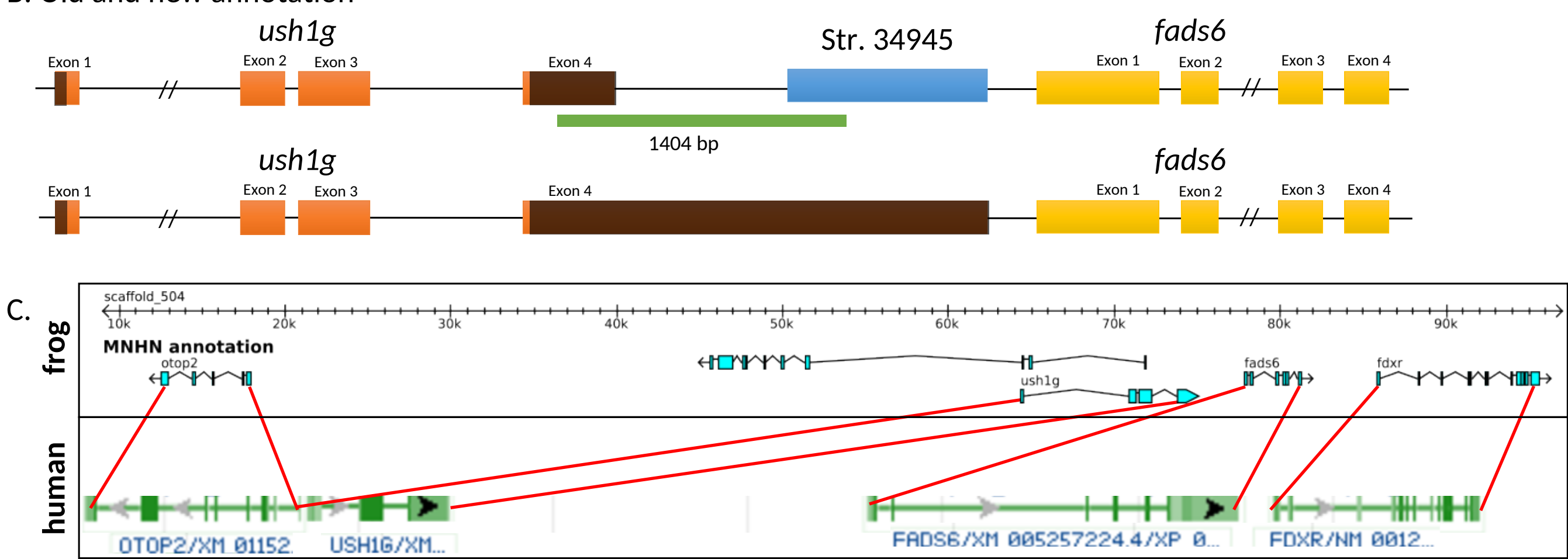